\title{
The Application of Multimedia Intelligent Rice Production System
}

\author{
Yao Yuxia, Wang Kunhao \\ The Information Engineering College of Changchun University of Science and Technology, Ji Lin \\ Chang Chun 130600 China \\ yx--yao@163.com; 1225449801@qq.com
}

Keywords: rice cultivation expert system, intelligent technology

\begin{abstract}
In order to realize the intelligent scientific farming, multimedia rice production intelligence system was developed. The study to the water in the rice planting, fertilizer, soil, pests and diseases, weeds and other field management as the breakthrough point, using computer technology to realize the man-machine interface of expert system, knowledge acquisition, inference engine, explain mechanism, etc., through the platform development, effective sharing of data, information, data, improve the analysis to identify various states and insect and pest occurrence and development of ability, effectively solve the various problems encountered in rice cultivation, in the field of rice cultivation management, agricultural services has important guiding significance and broad application prospects.
\end{abstract}

\section{Introduction}

In the long-term research and production practice, the agricultural science and technology personnel and managers although on rice production, management of agricultural production has made a number of scientific research achievements and accumulated rich experience. But due to the vast area of agricultural production in our country, the market, the scale of production sizes, and highly fragmented; High-level agricultural experts, low cultural quality of farmer science and technology; Science and technology communication is difficult, the agricultural production development level is very uneven. Especially with the development of information technology and intelligent agriculture in many agricultural information technology, represented by the expert system of the role of agricultural intelligent information technology is very outstanding, intelligent agriculture and digital agriculture in jilin province in the country and has not been in full swing ${ }^{[1]}$. Therefore, in order to make the agriculture modernization, intelligent information management network, scientific farming to better service for agriculture, rural areas and farmers, to make our country's agricultural production management and international standards as soon as possible, make the high quality and high yield of rice, development and application of multimedia expert system for rice production is very necessary, it is welcomed by farmers "rural experts don't walk". With "industry nurturing agriculture and cities support the countryside, application oriented, pragmatic, actively promote and improve efficiency of reason", speed up agricultural scientific and technological progress, improving agricultural comprehensive production capacity as a major and urgent strategic task in our country, the countryside is to agricultural information, agricultural intelligent driving rural modernization, play to the agricultural information and agricultural modernization "stack effect" and "multiplier effect", the "three rural" demand of information service, information service platform construction, science and technology advisory services, network technology combined with a variety of conventional media, model, resource sharing, promote information poverty alleviation, rural "digital divide" ${ }^{2]}$.

\section{The basic structure of a system}

Intelligent agricultural expert system is the main characteristic of massively parallel processing, distributed storage of information, direct input sample and the global collective action. The morphological parameters, data interface and reasoning mechanism, the output characteristics and 
diagnosis $^{[3]}$. In this system, knowledge can be extracted from the sample by computer, the expert knowledge, experience and reasoning for solving practical problems, through the computer to express. Basic system mainly has five parts, respectively for the agricultural knowledge base, agricultural database, reasoning mechanism, the user interface, and guide the agricultural production and so on. The technology using digital cameras and digital video camera to the subjects, the image and text input computer, using the research and development of shape recognition diagnosis system for processing, analysis, diagnosis and to obtain the morphological characteristics of the diagnosis, it is concluded that the corresponding measures to guide agricultural production ${ }^{[4]}$. Intelligent agriculture expert system process is shown in figure 1.

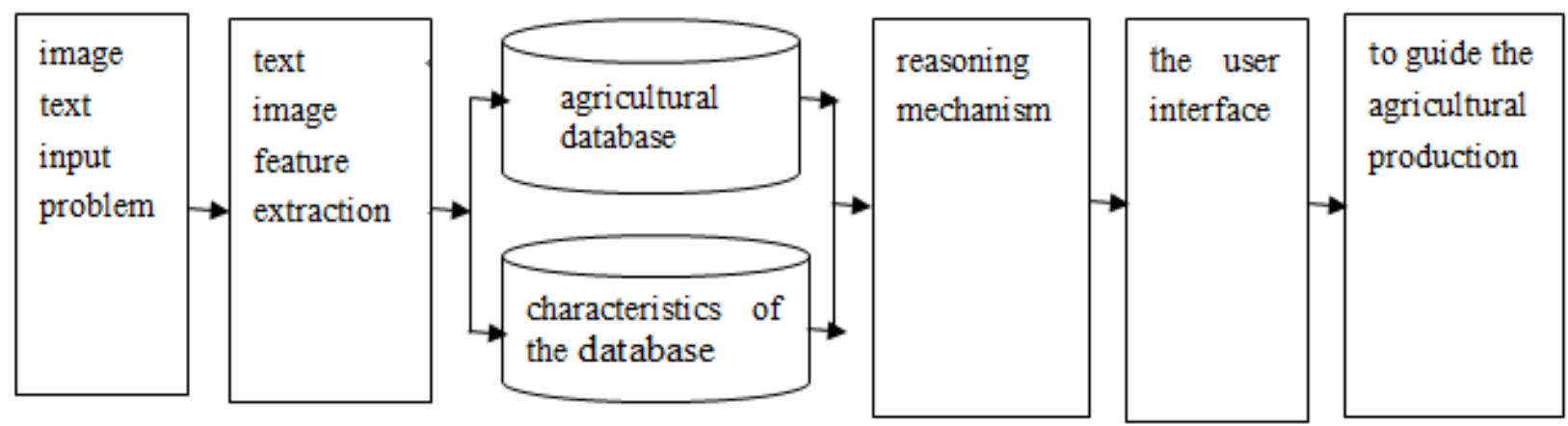

Figure 1 intelligence agriculture expert system process

Feature extraction: after essence is the user questions (can be text, also may be images), module for extracting image or text, respectively, by the soil, fertilizer and water conditions and plant root, stem and leaf length, size, leaf age, leaf color, disease and insect pests such as morphological characteristics and information, etc.

Agricultural knowledge base, is a collection of solving the issue of agriculture, including basic facts, rules and other relevant information, the library knowledge from domain experts, is the key to the ability of expert system, is the core part of agricultural expert system.

Agricultural databases: is agriculture based data collection, including production conditions, environmental conditions, such as reasoning mechanism is mainly composed of the scheduler and interpreter, is the core of the implementation of problem solving actuators ${ }^{[5]}$. (agricultural knowledge acquisition mechanism, is to solve the problem of agricultural experts from various specialized knowledge in the mind, or other tacit knowledge conversion to the knowledge base in various ways in there). Data mining is an effective auxiliary means of traditional knowledge acquisition, including the explanation mechanism, is to answer customer consultation, problem solving process to users or solving state institutions provide instructions.

User interface: it is between expert system and the user interaction medium of communication and information exchange. "Multimedia rice production intelligence system" of the main page as in figure 2 .

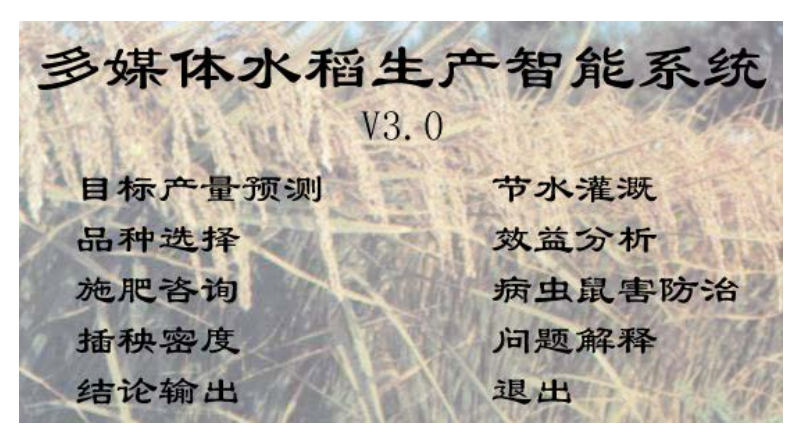

Figure 2 The main page of the system

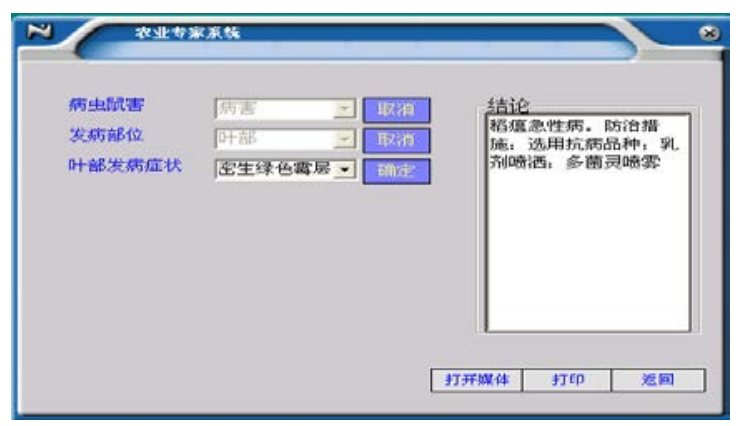

Figure 3. Reasoning and prevention

\section{The agricultural expert system design technology}

Experts in the field of choice: according to actual production situation in the region, select a representative and demonstration, and has good economic efficiency and the scale of production, at 
the same time to choose theory combined with production practice, the practicability of domain experts, can theoretically and actually to solve the actual problems existing in the production.

Expert system design: first, starting from the actual production to find out the main problems existing in the current rice production, according to the different problems, expert design system framework, main function modules and the problems need to be solved after, will each functional module, for better comprehensive analysis and diagnosis to lay a foundation for further work. User by clicking the corresponding link, but also can see all kinds of detailed knowledge base material, as a practical expert system, knowledge and data update in time, with the continuous update of data, knowledge will change accordingly. Main source of knowledge and the region, experts in the field of this, assisted with the experimental data, and technological achievements, such as data mining. All kinds of knowledge after modified perfect agricultural experts, sorting, discussed repeatedly, modifying, the knowledge of the rules. Regulation method is to determine the module first, and then determine the project decision, to determine the premise, the last write rules.

Method and technical route, data collection and knowledge acquisition is the key of the system is being developed, to develop rich in content, theme is distinct, data accurate "multimedia rice production intelligence system", must by authoritative experts in the field of knowledge, results, and on the basis of practical experience with a large number of documents and data as the supplement, at the same time to further the research base, in order to improve the system of scientific and practical. On this basis, the system overall design, text and image acquisition Production technology on the basis of advanced computer technology, the text description of present situation, characteristics, solve method is made into a form suitable for computer operation, such as complete expression of the interactive page design and media design .

System covers rice production target, species selection, fertilization, planting density, water-saving irrigation, pest prevention and control of diseases and pests, problem solving, benefit analysis, the conclusion output, etc. 8 module system. The system has the interface is beautiful, clear image, convenient maintenance, good interaction and the characteristics of strong practicability, easy to the general managers of agricultural production, agricultural technology popularization personnel. In order to guarantee the accuracy of the software and practical, extensively solicit expert users, agricultural science and technology workers and peasants, especially with the unceasing change of environmental factors and production conditions, the deepening of the research in this field, and new technology and new knowledge constantly emerging, the system needs to constantly adjust, regularly evaluate the logical syntax in the knowledge base, including the knowledge of the consistency and integrity, prompt errors in the error message the type, nature and location, edit and modify the definition of knowledge rules. Through the feedback, found problems in the experiment and demonstration, to modify and perfect the system in time, to adapt to the need of actual production ${ }^{[6]}$.

"Multimedia rice production intelligence system" have in common, compared with R/WCSODS has innovation point. Its innovation point lies in has stronger practicability and the ability to deal with incomplete information and data, the rice variety selection, production forecast, irrigation, fertilization, cultivation, pest and disease diagnosis, prevention, etc., using the computer technology and the method of combining a variety of software developed with network, expert system of components, intelligent, hierarchical and diversified characteristics. The system to select the water in the rice planting, fertilizer, soil, pests and diseases, weeds and other field management as the breakthrough point, using computer technology to realize the man-machine interface of expert system, knowledge acquisition, inference engine, explain mechanism, etc., through the platform development, effective sharing of data, information, data, improve the analysis to identify various states and insect and pest occurrence and development of the ability of combining rich improve diagnosis technology, expert system in guiding agricultural production to get extensive application]. At present, although based on computer network of agricultural information technology service system has been initially formed, across the country have set up their own information network, but overall, as a result of the limitation of technology, the existing network application layer has mostly stay in focus on agricultural materials information, production information and practical technology 
on the release of the lack of because of appropriate decision-making function, and the strain of complicated and open multimedia agriculture expert system, agriculture is difficult to through the network and pertinence of the agricultural industry management information about the dynamic ${ }^{\text {[7] }}$. With the continuous development of information technology, through the network should be able to achieve remote direct access to a large database and the host system software resource sharing, timely access to the latest technical information and market dynamics. In order to promote the research, application and promotion of agricultural intelligent system, the state ministry of science and technology to agricultural intelligent information technology application demonstration project listed as national 863 project support, [8]the national of agricultural intelligent information technology application in the promotion, raise the contribution rate of science and technology in agriculture, accelerate the transformation of scientific and technological achievements of agricultural expert system engineering is of great significance.

\section{Rice production is the application of multimedia intelligent system}

Aiming at the problems above, has now developed the represented by "multimedia rice production intelligence system" of eight expert system, and obtained the national copyright administration software copyright registration, is popularized and applied in multiple demonstration area. The practice shows that the expert system for demonstration area of peasant household production of rice varieties selection, prediction, irrigation, fertilization, cultivation, pest and disease diagnosis, prevention and cure of popularization and application, has played a very good guidance role in crop production, especially the demand of information service platform construction information, visualization and network, strengthen the function of computer interface interaction, simplifies the application, use very convenient, is popular among farmers, through the application of the system, changed the traditional cultivation techniques for a long time in a partial, scattered, single and passive situation. Both improve the comprehensive quality of the farmers' scientific farming and management, and increase the economic income. Now, with the development of science and technology and the modernization and information, the improvement in the recognition of digital level of scientific farming, in the rural areas there are a lot of planting large, breeding farmer who have bought the computer, the application and popularization of agricultural expert system provides a favorable conditions, also can undertake village, groups, families, and other forms of training and to popularize and apply; At the same time, the system can be used as the spark network plans and experts compound training textbooks, also can be used as agricultural colleges and universities graduate students, undergraduates of auxiliary materials ${ }^{[8]}$.

To sum up, through the demand of the characteristics of the large area of farmland investigation, analysis, evaluation, application, modify and maintain, on the basis of this system, developed suitable for different problems in the field of high availability, reliability, and extensible expert system, to facilitate the promotion of the technological achievements, to improve grass-roots agricultural researchers and producers of information consciousness of science and technology and scientific management level, improve agricultural expert system for production efficiency, effectiveness, efficiency, construction of resource saving, the green economy, circular economy, promoting the transformation of technological achievements, talent of education informatization, informatization policy, regulations and standards is of great practical application value and broad application prospects.

\section{Reference}

[1] Yao yu-xia, Chen Gui-fen, Si Xiu-li, Application of multimedia ginseng producing agriculture intelligent expert system to cultivating of ginseng, [J]. Chinese Traditionl and herbal drugs, 2004.5.(588-590)

[2] Liu Gan -na, Multi-media application foundation[M] .higher education publishing house, 2003.12.(85-137). 
[3] Zheng Guo-qing, Zhang Rui-ling, Gao Liang-zhi,Progress and perspective in maize growth simulation model in China Maize Science), [J].Corn science 2,003. 2.(66-70).

[4] Shen Zuo-rui, Qiao Xiao-feng,Wang Zhonh-hui,Farm crop plant diseases and insect pests prevention and cure the long range consult the development of the service system and relevant of policy suggestion), [J].The compute and agriculture), 2003.4.(14-16).

[5] Gao Liang-Zhi, Jin Zhi-qing, Huang Yao, RCS0DS). (Rice Cultivational SimulationOptimization-Decision-Making System(RCSODS).BeiJing: China Agriculture Press), [M] Chinese agriculture publishing house)1992(1-110).

[6] Yao Yux-ia, Chen Gui-fen, Hou Yuan-chun, Intelligent expert system of morphological diagnosis for rice plant diseases and insect pests [J].Journal of jilin university(Information science edition)2003, 4(378-381)

[7] Yao yu-xia, The Research and Application of Remote Sensing Technology in MaizeYield Estimation of Jilin, 2014IEEE Workshaop on Advanced Research and Technology Industry Applications(WARTIA) OTTAWA CANADA 2014.9(215-218)

[8] Cai jia-pei, Application of Computer Technology in Maize Production Implementation Precision Agriculture,Selected peer reviewed papers from the $20144^{\text {th }}$ international Conference on intelligent System and Applied Material (GSAM 2014) August 23-24,2014,Taiyuan,China. 2014.11 (2153-2156) 